\title{
Computer-Assisted Analysis of Human Semen Concentration and Motility
}

\author{
Karima Boumaza, Université des Sciences et de la Technologie d'Oran, Algeria \\ (iD) https://orcid.org/0000-0002-8623-0477 \\ Abdelhamid Loukil, Université des Sciences et de la Technologie d'Oran, Algeria
}

\begin{abstract}
Computer-assisted semen analysis systems insist on evaluating sperm characteristics. These systems afford capacity to study and evaluate sperm statistical and morphological characteristics such as concentration, morphology, and motility, which have an important role in diagnosis and treatment of male infertility. In this paper, the proposed algorithm allows the assessment of concentration and motility rate of sperms in microscopic videos. First, enhancement process is required because of microscopic images limitations such as low contrast and noises. Then, for true sperm recognition among noise and debris, a hybrid approach is proposed using a combination between segmentation techniques. After, the use of geometric features of the bounding ellipse of the sperm head led to define sperm concentration. Finally, inter-frame difference is applied for motile sperm detection. The proposed method was tested on microscopic videos of human semen; the performance of this method is analyzed in terms of speed, accuracy, and complexity. Obtained results during the experiments are very promising compared with those obtained by the traditional assessment, which is the most widely used and approved in the laboratories.
\end{abstract}

\section{KEYWORDS}

Bounding Ellipse, CASA, Concentration, Motility, Semen Analysis, Watershed Segmentation

\section{INTRODUCTION}

For diagnosing male infertility, a sperm assessment is necessary as an initial and essential step. Among the most important parameters, we can cite sperm concentration (or density), sperm motility (or vitality), and sperm morphology (Nallella, et al., 2006). According to the World Health Organization (WHO), 15\% of couples in the world suffer from infertility. Males are found to be solely responsible for $20-30 \%$ of infertility cases and contribute to $50 \%$ of cases overall. The evaluation of sperm parameters in fertility clinics and laboratories is analyzed manually by andrologist technicians. Visual problems and fatigue of the human operator negatively affect the results and make them subjective, leading to considerable intra and inter laboratory variability.

Since the 1980s, these analyzes have been performed using automatic systems called "Computer Assisted Semen Analysis" (CASA) (Amann, R. P., et al., 2014). The processing microscopic images 
in a computer can be useful to extract quantitative information about the specimen. The poor quality of sperm microscopic videos and the collisions of spermatozoids on the "blade + lamella" walls make CASA systems suffer from certain limitations and up today, evaluation of sperm parameters are not yet repeatable and objective . For that the microscopic images used are processed by using digital image processing techniques as enhancement techniques, segmentation techniques, object measurement, object classification,....etc.

The improvement of sperm analysis by CASA systems is currently a hot topic research. Several studies have been carried out for the automated sperm detection (Syahputra et al., 2018). In 2010, authors in (Abbiramy et al., 2010) proposed a technique for sperm motility detection which is based on Laplacian filter and Median filter as preprocessing step. Then, they segmented the images using simple thresholding and morphological operation with labeling process. The differential method is applied for reducing complexity and faster tracking of spermatozoids. One year after, Mahdavi et al. (Mahdavi et al., 2011) have thought to benefit from the elliptical shape of the spermatozoid head to search on the image that blobs having this form. Simple thresholding has been done to create a binary image from the denoised image. The threshold value is chosen after several experimental tests. The elimination of badly classified pixels is performed by morphological operators. To reinforce the detection of true spermatozoa, the authors used the geometric characteristics of labeled objects as the ratio between the major axis and the minor axis of the ellipse to eliminate false sperm.

In 2017, authors in (Urbano et al., 2017), in order to detect a spermatozoid in an image, have applied a Gaussian filter for smoothing the noisy image then used Laplacien of Gaussian filter (LoG) for detecting contours. The binary segmented image was obtained by using Otsu's thresholding method (Otsu, 1979) followed by many morphological operations. At the end, each object is labeled so they can separate sperm from non-sperm with calculating the centroid coordinates and sperm dimensions. By the year 2018, the authors in (Khalifa et al., 2018) proposed a system for counting and tracking sperms in videos. First, they have used images in HSV domain. Then thresholding process is applied. After the next steps insist to grouping pixels and then accept and reject pixel groups according to a pixel group value to remove noise and an unwanted group of pixels. After that, it calculated the center of the pixel groups and counts all pixel groups which indicated the number of sperm. Drawing a rectangle around the pixel groups is done in final step for tracking purposes.

These works based on thresholding techniques for image segmentation which are efficient approaches to sperm cells image but with highly sensitive performance to threshold values. In other words, slight errors in selecting the optimal threshold may lead to either significant lack of sperms or increased false detections.

In 2011, authors in (Ravanfar et al., 2011) applied Median Filter and Watershed Segmentation technique to detect sperm in low contrast image. The particle filter is then used to obtain object tracking. Watershed segmentation is one of region based segmentation which is sensitive to the distance between the sperm cells, they are not suitable for highly concentrated samples. Authors in (Bijar et al., 2012) used a simple threshold to build a mask containing all candidate pixels which may belong to the sperm's head and Mid-piece. Then, they used a hybrid method to create a denoised image. The nucleus and Mid-piece are segmented through a method based on a Bayesian classifier which utilizes the entropy based expectation-maximization (EM) algorithm and Markov random field (MRF) model. Artifacts and debris in images affect the performance of such methods, which will be a source of error.

In 2016, authors in (Shaker et al., 2016) proposed a new technique for spermatozoa head and tail detection. For head detection, they used a combination between Canny Edge Detector and Gradient Vector Flow (GVF) active contour and for the tail; they used curvature and edge energy. However, extracting correct contours requires extensive computation. This renders contour-based algorithms very slow and unsuitable for pragmatic situations. For Reza Arkanfari,(Arkanfari et al., 2018) a new method was introduced for detecting sperms in microscopy images, it based on the estimation of Zernike moments followed by spatial processing proposed by (Shojaedini et al., 2017). The proposed 
method includes two steps. In the first step, Zernike moments are estimated to select candidate pixels belonging to sperm cells. In the second step, the correct sperms are confirmed by using the connectivity concept and morphological operations. The proposed method has a problem to define the sperm from debris when they have the similar shape and size.

All the previously discussed techniques have been confronted with several problems that can be summarized in the confusion between a real spermatozoon and debris with similar size which will distort the results; Poor adaptability of the technique according to the quality of the sperm samples lighting.

In order to improve the results achieved by each of these techniques, the work presented in this paper proposes a new algorithm for both detection, counting and motility assessment of sperm cells, to achieve higher accuracy and more robust performance in laboratorial sperm cells video streams with different particle densities. First, it focuses on the process of segmentation, where we have introduced a new method based on the combination of watershed segmentation and sobel filter. This detect and identify sperm cells forms debris and improves the shapes of it in the images. For the motility, we have used the three frame differencing algorithm; this algorithm may detect mobile sperm with a simple calculation and avoid the problem of the sperm tails movement which cause a difficulties in the identification of the mobile ones.

The remaining part of this paper is organized as follows: in section 2; the functional architecture of the proposed system is developed. Section 3 presents the experimental results and their discussions. Finally, in section 4, we conclude the study and then propose future improvements.

\section{FUNCTIONAL ARCHITECTURE OF THE PROPOSED SYSTEM}

The sperm motility and concentration assessment in this research consists of several steps that begin with the preprocessing step which start by an $\mathrm{YCbCr}$ process on the human sperm microscope image to obtain Gray level of image (Y Channel). Furthermore, Gaussian process is done to smooth the images. Noises reduction in the images is done by 2-D Discrete Wavelet Transform. The preprocessing process is followed by the crucial and important process which is segmentation process; it aims to separate spermatozoas from other impurities (debris, seminal fluid, noise). It proceeds by the reinforcement of two segmentation techniques: the first is region-based called "Watershed Segmentation" and the other is contour-based called "Sobel Edge Detector". As a result of this process, we obtain a binary image containing white blobs of different sizes. In order to keep only the spermatozoas, a thresholding on the blob surface is performed. The labeling process is done for the numbering and counting the objects present in each sperm microscope image, the bounding box process is done to recognize the object in each image of the sperm microscope, and the classification using the elliptical shape and the size value obtained by an expert. The result obtained at the output of this module is the total number of spermatozoa which means the concentration. The last process in our proposed technique insists to separate motile spermatozoas from immotile ones by the difference of three successive frames method. After completing all steps, with a simple labeling and counting of blobs, sperm concentration and motility values are assessed. The methodology of this research can be seen in the general architecture at Figure 1.

\section{Pre-Processing Module}

Sperm microscopic videos at the input of the analysis process are affected by a random noise resulting from the acquisition process. Added to this, the lighting used is non-uniform and may vary from one sequence to another. To remedy these flaws, we have been partially inspired by the work in (Ghasemian, F. et al., 2015). The first step is to convert the RGB color space, in which the raw video is encoded, to the $\mathrm{YCbCr}$ space, which is known to be very robust against lighting changes. The second step concerns a 2D Gaussian filtering, and the third step consists of filtering in the timefrequency domain by discrete wavelets: 
Figure 1. Functional architecture of the proposed system

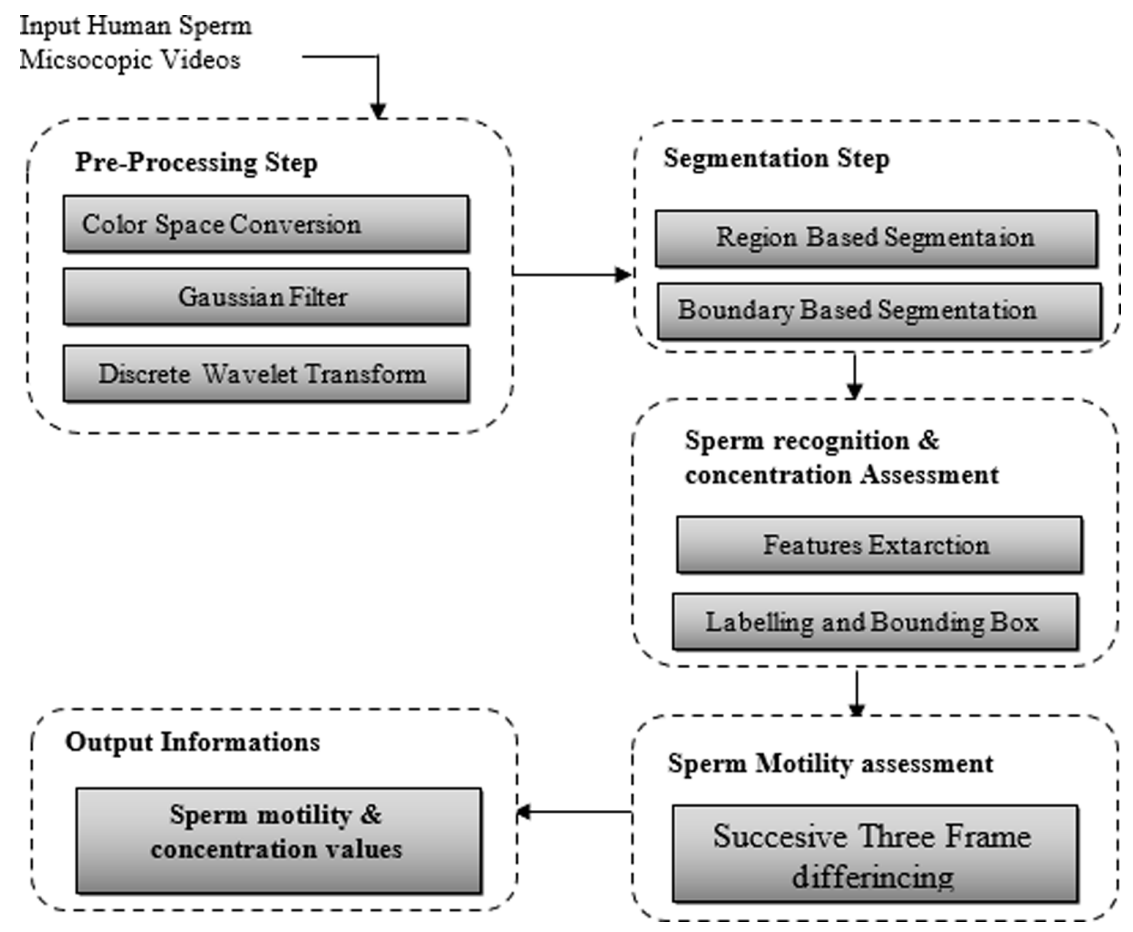

\section{Converting RGB Space to $\mathrm{YCbCr}$ Space}

Sperm microscopic videos are coded in the RGB space, clocked at 25 frames per second and having a resolution of $240 \times 320$ pixels. To separate the luminance component from the chrominance component, we converted RGB space to YCbCr by using Equation (1):

$$
\left[\begin{array}{c}
\mathrm{Y} \\
\mathrm{Cb} \\
\mathrm{Cr}
\end{array}\right]=\left[\begin{array}{c}
16 \\
128 \\
128
\end{array}\right]+\left[\begin{array}{ccc}
+65.481 & +128.553 & +24.966 \\
-37.797 & -74.203 & +112.000 \\
+112.00 . & -93.786 & -18.214
\end{array}\right]\left[\begin{array}{c}
\mathrm{R} \\
\mathrm{G} \\
\mathrm{B}
\end{array}\right]
$$

From the $\mathrm{YCbCr}$ color image obtained, we selected the Y component alone which strongly looks-like the corresponding gray-level image.

\section{Smoothing with 2D Gaussian Filter}

To reduce the noise, we applied a 2D Gaussian filter given by Equation (2):

$$
g(x, y)=\frac{1}{2 \dot{A}^{2}} e^{-\frac{\left(x^{2}+y^{2}\right)}{2 \tilde{A}^{2}}}
$$

where $\sigma$ is the standard deviation of the Gaussian distribution and $(\mathrm{x}, \mathrm{y})$ are pixel coordinates in the $2 \mathrm{D}$ image. After many experience the highest smoothing rate is marked with $\sigma=0.9$ and kernel $3 \times 3$. 


\section{Filtering with Discrete Wavelet Transform}

Wavelets have recently been considered as a very powerful tool for the denoising of medical images because they face a problem of noise components located in high frequencies. The discrete wavelet maps an image into a set of coefficients that constitutes a multi-scale representation (Grover, T., 2016),(Mustafa, N. et al., 2014).after many experience, in the use of discrete wavelet "Coifflet" limited at 04 levels of decomposition (4 octaves) we could have removed noise with an acceptable rate.

\section{Sperm Segmentation Module}

At this module input, the images are denoised and more contrasted. They contain spermatozoas (region of interest - ROI) but in addition, they include other impurities (Background - BKG).

The segmentation process intends to make separation between the ROI and the BKG. To achieve this goal, the adopted technique proceeds by strengthening two segmentation techniques (see Figure 2): The first is region-based called "watershed segmentation" and the other is boundary-based called "Sobel edge detector ". The image at the entrance of this module will therefore go through two competing treatments as follows:

- Median filtering (3 x 3) to suppress impulse noise followed by a Sobel edge detector(Gao,2010). As a result, we get a contours image.

- Watershed segmentation to obtain uniform regions. From these blobs, we extract the centroids.

At the downstream of these two treatments, we make the projection of the centroid of each blob on its contour so that finally we obtain the ROI with its contours in white color and the BKG in black color.

The image obtained at the end of this phase, shows the presence of objects with similar size as spermatozoid, and others having sizes higher or lower. A thresholding by blobs size allows keeping only blobs of size close to that of a spermatozoid.

\section{SPERM RECOGNITION AND CONCENTRATION ESTIMATION MODULE}

The second module output image is binary and includes blobs having similar sizes to spermatozoa's but with various shapes. To leave only real spermatozoas, this module has for role to identify them and eliminate the other objects. The adopted solution lies in the exploitation of the elliptical form of the spermatozoid's head as well as its surface. To do this, we calculate for each blob the characteristics

Figure 2. Sperm segmentation organigram

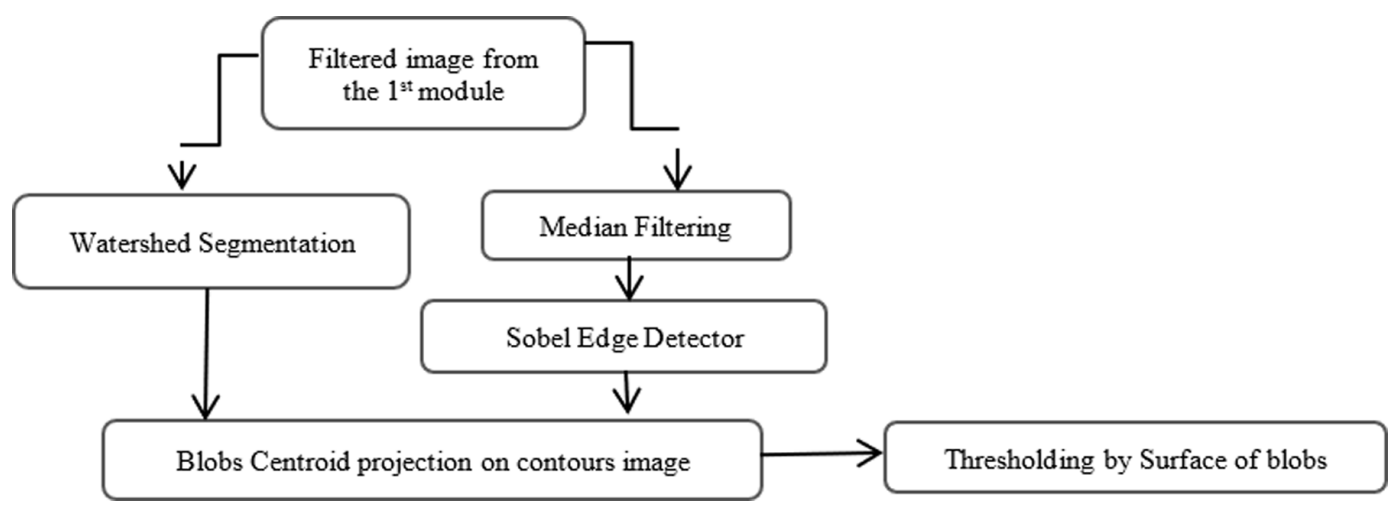


of the bounding ellipse and its surface (see Figure 3). Then we take as discriminating characteristics vector values of:

- The major axis 'a' of the ellipse (in pixels):

- The minor axis ' $b$ ' of the ellipse (in pixels);

- The surface ' $S$ ' of the blob (in pixels);

Figure 3. Ellipse geometry

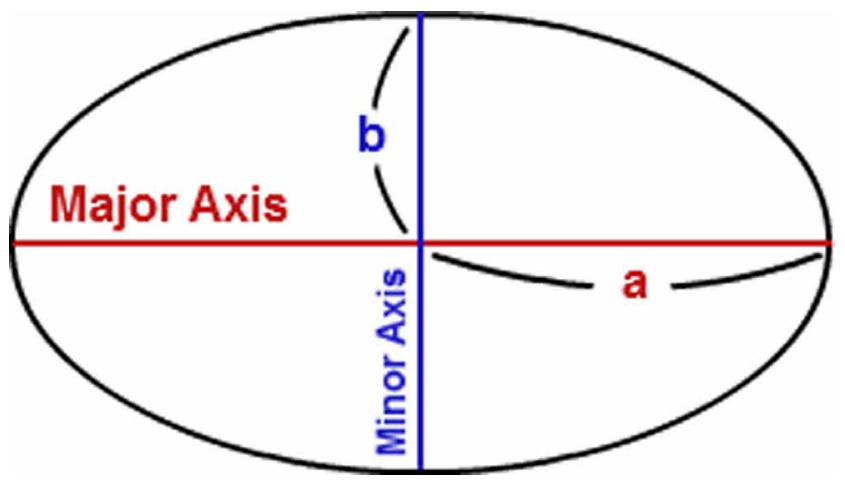

As preliminary work of the classification step, we manually analyzed, with the help of andrologist technicians, several video sequences of our database and manually measured the limits of the 3 characteristics for spermatozoas. The experimental values found are:

- The major axis limits value: $a \in[4.20,16.90]$ pixels

- The minor axis limits value: $b<7.74$ pixels ;

- The area limits value: $S \in[55,200]$ pixels ;

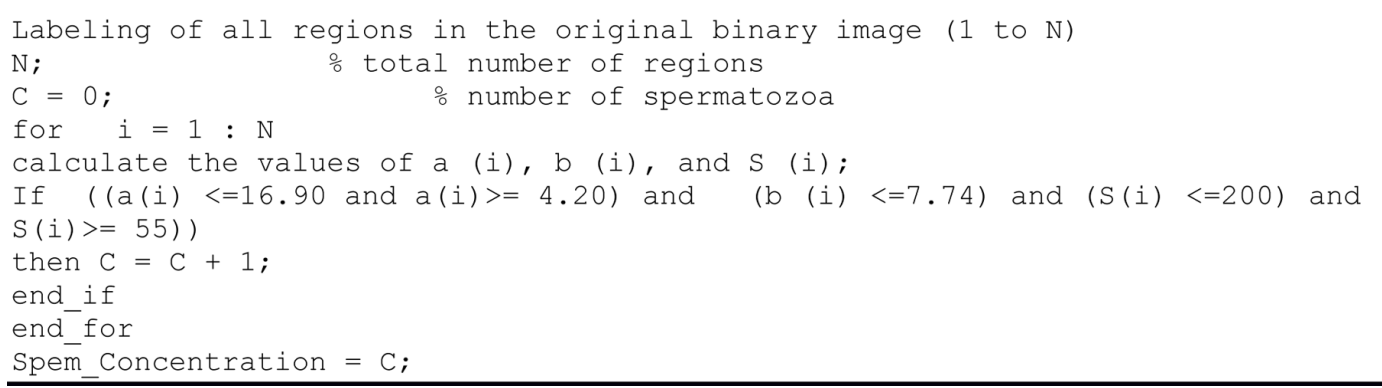

After that, we applied on this characteristic vector the treatment cited in 'Algorithm_1'. At the end of this module, we pass the output image in a labeling and counting process to get the concentration value. 


\section{MOTILE SPERM DETECTION MODULE}

The result of the previous step is a binary image in which the objects of interest (spermatozoas) are in white color (1) and the background in black color (0). In order to detect only motile spermatozoa, we apply the inter-frame differences technique between 3 successive binary images "Ik-1, Ik and $\mathrm{Ik}+1$ " for the whole of the video sequence. The principle of this motion segmentation method is as follows (Gang, L. et al., 2013) (see Figure 4):

Figure 4. Motile sperm detection using three frames differencing technique

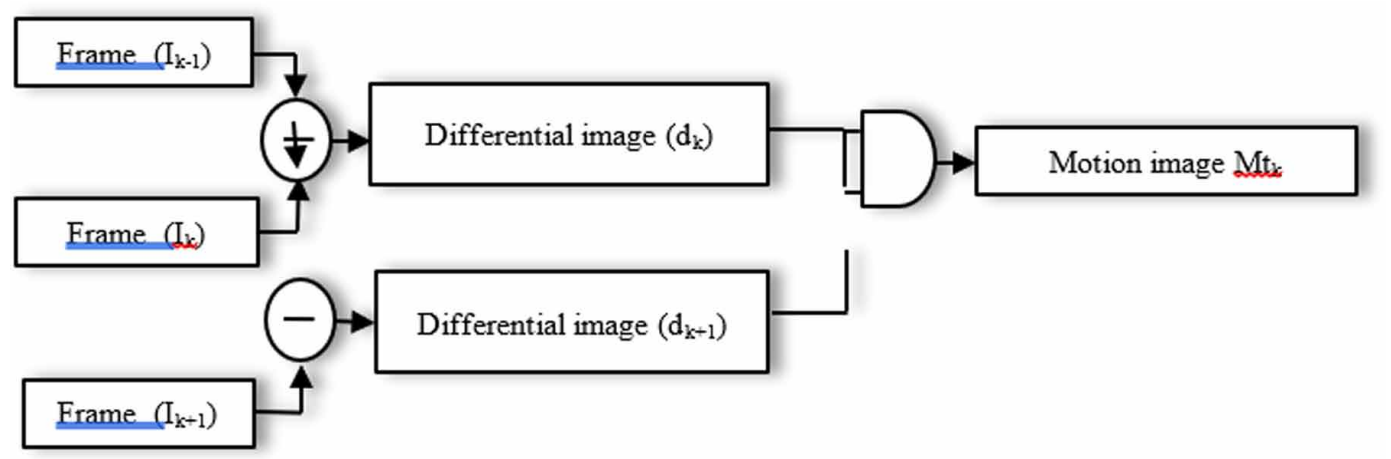

Calculate the two differential images between the 3 successive frames $\mathrm{dk}$ and $\mathrm{dk}+1 \mathrm{using}$ Equations (3) and (4):

$$
\begin{aligned}
& \mathrm{d}_{\mathrm{k}}=\mathrm{I}_{\mathrm{k}}-\mathrm{I}_{\mathrm{k}-1} \\
& \mathrm{~d}_{\mathrm{k}+1}=\mathrm{I}_{\mathrm{k}+1}-\mathrm{I}_{\mathrm{k}}
\end{aligned}
$$

Obtain a binary segmentation image of the motion Mtk by computing the logical operator 'AND' between the two previous differential images dk and dk+1 as in Equation (5):

$$
M t_{k}=\left(d_{k}\right) A N D\left(d_{k+1}\right)
$$

The motility rate is obtained by labeling and counting all white blobs in the motion image Mtk.

\section{RESULTS AND DISCUSSION}

For the experimental evaluation, we have at our disposal a public database with a microscopic videos of sperm samples provided to us by the Fertility and Infertility Center of Isfahan FICI - Iran (Imani, Y. et al., 2014). The sperm samples belong to 30 patients, and are filmed by a phase-contrast electron microscope with a " $\times 120$ " magnification (zoom), a resolution of ' $240 \times 320$ ' pixels and clocked at 25 frames/sec. It should be noted that all WHO directives and instructions have been respected for capturing these videos (Cooper et al. 2010). The software implementation was made on a computer equipped with a Core i5-2.4 GHz processor and $8 \mathrm{~GB}$ of memory. The used software implementation is MATLAB-2016b with its toolbox "Image Processing Toolbox - IPT". For a first evaluation of our 
system, we randomly selected a series of 8 video sequences of spermatozoa belonging to different patients showing the 4 types of motility as defined by WHO and with different densities semen. The selected videos were first processed manually by a three experts to obtain a ground truth to compare the proposed method with others implemented methods. In what follows, we present the experimental results obtained for each of the 4 modules:

\section{Pre-Processing Module}

As described in section 2.1, preprocessing of raw images consists of converting the color space and reducing noises. The experimental results are shown in Figure 5: top row is the original video frame of the motile sperm, the next row is the conversion to $\mathrm{YCbCr}$ color space. Then we selected only the Y component for further.

Figure 5. (a, a', a") Raw RGB input images (b, b', b') Only 'Y' component of the YCbCr image (c, c', c') Gaussian filtered image (d, d', d") Discrete Wavelet filtered image.

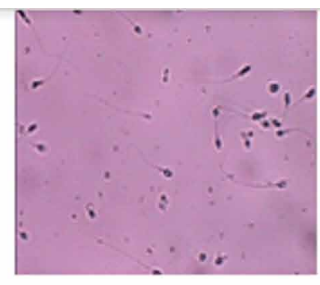

(a)

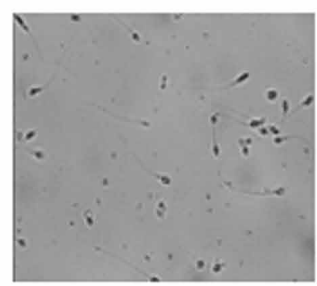

(b)

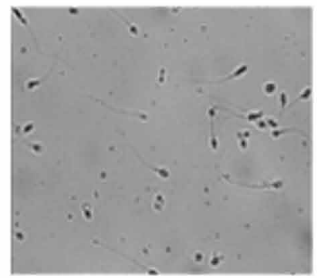

(c)

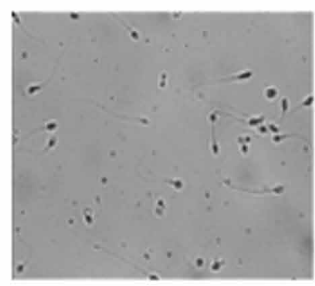

(d)

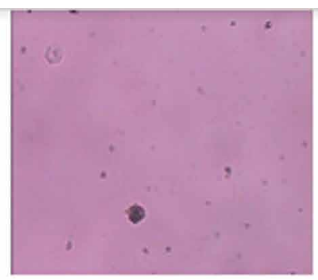

(a)

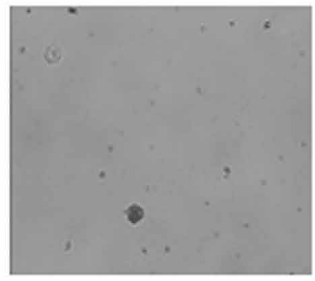

(b')

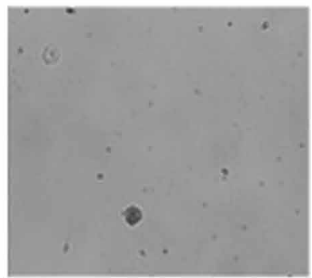

(c)

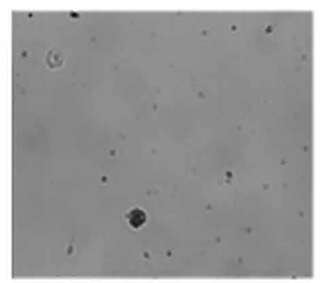

(d)

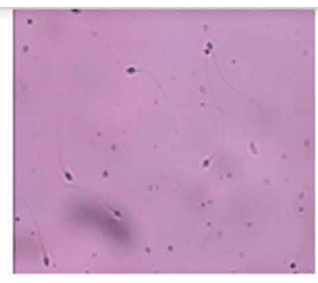

(a")

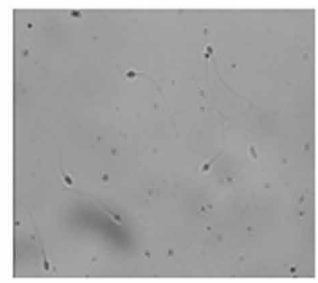

(b)

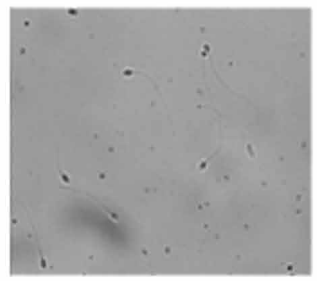

(c")

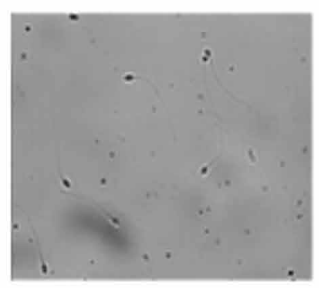

(d) 
processing. The noise reduction is done in two steps: a Gaussian filtering mask $(3 \times 3)$ with a standard deviation of ' 0.9 ' is applied (the third row), then a Discrete Wavelet Filtering up to the fourth decade is carried out. In last row, we show the resulting denoised image that contains spermatozoids as well as other unwanted objects (debris).

\section{SPERM SEGMENTATION MODULE}

As explained in section 2.2, sperm segmentation begins by filtering the resulting image of the first module. Indeed, in the experimental part, we want to show that the best filter to apply to this kind of images is the median filter ( $3 \times 3)$. Table 1 compares 3 kinds of smoothing filters: the median filter $(3 \times 3)$, the nearest symmetric neighbor filter $(\mathrm{SNN})$, and the averaging $(3 \times 3)$ filter. The metrics used to choose between the 3 filters is Peak Signal to Noise Ratio (PNSR). From results in Table 1, the median filter gives the best results (higher PSNR) and the filtered image is shown in Figure 7 in the first row.

Table 1. PSNR value obtained for the 3 types of filtering

\begin{tabular}{|l|l|l|l|}
\hline & \multicolumn{1}{|c|}{ Median Filtering (3x3) } & \multicolumn{1}{|c|}{ SNN Filtering (3x3) } & \multicolumn{1}{c|}{ Average filtering (3x3) } \\
\hline PNSR & 44.9808 & 44.7013 & 39.3218 \\
\hline
\end{tabular}

The next step is edge detection using the Sobel Edge Detector. The result is given in Figures 6.b, b', b'. Similarly, regional segmentation is carried out by applying Watershed technique. The result is shown in Figures 6.c, c', c", and finally, the binary image containing the spermatozoa is obtained by merging the two segmented images. The final result is shown in the last row in the Figure 6.

Then, sperms were detected by applying the proposed method (WS-SF), Watershed Segmentation (WS) and Sobel Contour Detector (SCO). Finally, the performance of each algorithm was determined by comparing its results with manual detection results.

The aforementioned objective measures were computed by evaluating the images resulted from the application of the algorithms on eight samples with different particles' densities in reference to experts marked sperm cells images.

The results of WS, SCO, and WS-SF algorithms for the sample images are presented in Table 2.

Based on the results, the following points are noteworthy. For all of sample images (except sample No. 1, 4 and 7 from the specificity point of view and sample 5 from accuracy and specificity) the proposed algorithm provides the best results for all metrics. For these samples, the specificity of WS-SF is slightly less than that of the WS and SCO . This is due to its slightly lower values of FP (detected sperm cells) and the high amount of TN (missed sperm cells).

The proper results of this segmentation algorithm for the 2nd samples are confirmed in Table 2. For all the implemented methods, the accuracy, precision, sensitivity, specificity and F-measure of this sample are the highest on average. For the 6th and 7th samples with high particle densities WS-SFalgorithm's accuracy, precision, sensitivity and F-measure are increased by $9 \%, 28 \%, 8 \%$, and $21 \%$, on average respectively, compared to those of the WS method.

The noteworthy point about the high amount of TN compared to FP, is that for the evaluation the segmentation step of this research based the results of different algorithms are compared with the results of the visual inspection of the experts as a ground-truth, and they are not considered all sperm-shaped objects as real sperm cells. 
Figure 6. (a, a', a") Median filtered image (b, b', b") Contours image by Sobel Detector (c, c', c") Segmented image by Watershed Algorithm (d, d', d") Sperm Region Detection

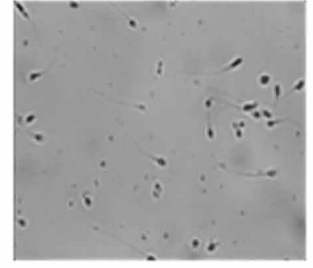

(a)

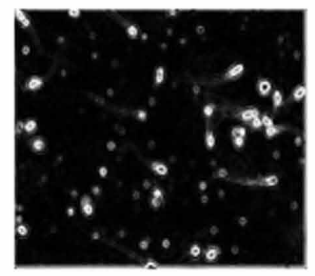

(b)

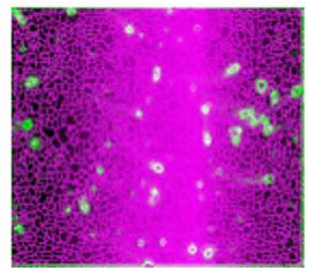

(c)

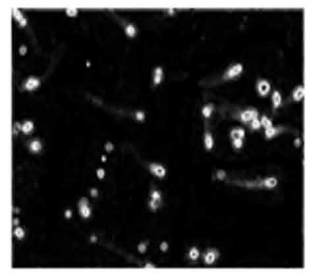

(d)

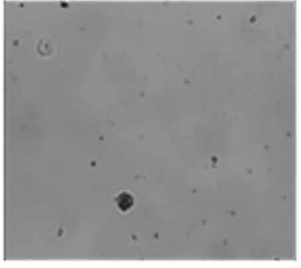

(a')

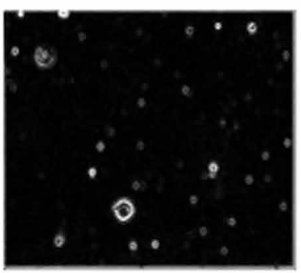

(b')

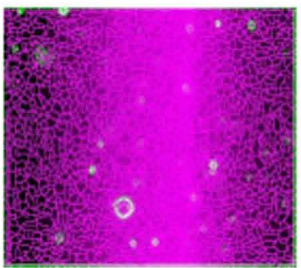

(c)

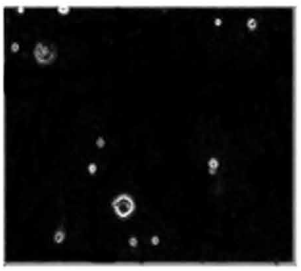

(d)

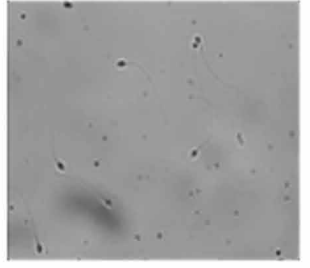

(a")

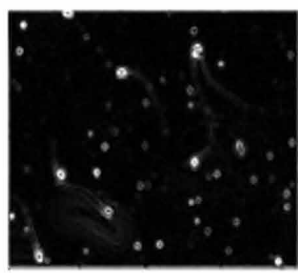

(b)

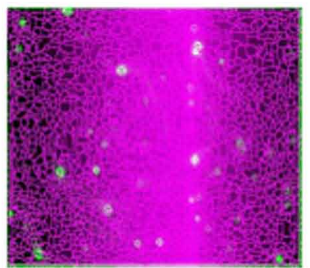

(c")

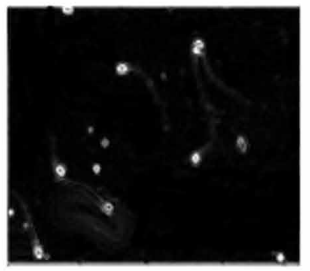

(d")

\section{Sperm Recognition and Concentration Estimation Module}

As explained in section 2.3, in Figure 7, the top row present the labeled blobs bounding ellipse drawn on each blob and in second row, the blobs classification is made. The concentration value is obtained by labeling then counting all blue blobs.

\section{Motile Sperm Detection Module}

As explained in section 2.4, the last step is the detection of motile spermatozoas. To do this, we apply the inter frame subtraction algorithm of 3 consecutive binary images as shown in Figure 4 and Equations (3), (4) and (5): In the Figure 8, the 3 images (a), (b) and (c) show the 3 successive frames $I_{k-1}, I_{k}$, and $I_{k+1}$ in the same video. The two images (d) and (e) of the same figure show the differential images $d_{k}$ and $d_{k+1}$. The resulting image (f) represents the motility image. It contains 
Table 2. Comparison of the results of different algorithms on sperm cells images

\begin{tabular}{|c|c|c|c|c|c|c|c|c|c|c|c|}
\hline $\begin{array}{l}\text { Videos } \\
\text { No }\end{array}$ & $\begin{array}{c}\text { Samples } \\
\text { No }\end{array}$ & Algorithm & $\mathbf{T P}$ & TN & FP & FN & $\begin{array}{c}\text { Accuracy } \\
(\%)\end{array}$ & $\begin{array}{c}\text { Presicion } \\
(\%)\end{array}$ & $\begin{array}{c}\text { Sensitivity } \\
(\%)\end{array}$ & $\underset{(\%)}{\text { F_mesure }}$ & $\begin{array}{c}\text { Specificity } \\
(\%)\end{array}$ \\
\hline \multirow{3}{*}{01} & \multirow{3}{*}{03} & WS & 2 & 15 & 6 & 1 & 70,83 & 25,00 & 66,67 & 36,36 & 71,43 \\
\hline & & SCO & 1 & 10 & 3 & 2 & 68,75 & 25,00 & 33,33 & 28,57 & 76,92 \\
\hline & & WS-SF & 3 & 9 & 3 & 1 & 75,00 & 50,00 & 75,00 & 60,00 & 75,00 \\
\hline \multirow{3}{*}{02} & \multirow{3}{*}{17} & WS & 13 & 20 & 15 & 4 & 63,46 & 46,43 & 76,47 & 57,78 & 57,14 \\
\hline & & SCO & 11 & 15 & 7 & 6 & 66,67 & 61,11 & 64,71 & 62,86 & 68,18 \\
\hline & & WS-SF & 16 & 13 & 5 & 1 & 82,86 & 76,19 & 94,12 & 84,21 & 72,22 \\
\hline \multirow{3}{*}{03} & \multirow{3}{*}{05} & WS & 2 & 14 & 9 & 3 & 57,14 & 18,18 & 40,00 & 25,00 & 60,87 \\
\hline & & SCO & 2 & 10 & 12 & 3 & 44,44 & 14,29 & 40,00 & 21,05 & 45,45 \\
\hline & & WS-SF & 5 & 9 & 5 & 1 & 70,00 & 50,00 & 83,33 & 62,50 & 64,29 \\
\hline \multirow{3}{*}{04} & \multirow{3}{*}{04} & WS & 2 & 15 & 12 & 2 & 54,84 & 14,29 & 50,00 & 22,22 & 55,56 \\
\hline & & SCO & 1 & 11 & 8 & 3 & 52,17 & 11,11 & 25,00 & 15,38 & 57,89 \\
\hline & & WS-SF & 4 & 5 & 6 & 1 & 56,25 & 40,00 & 80,00 & 53,33 & 45,45 \\
\hline \multirow{3}{*}{05} & \multirow{3}{*}{04} & WS & 3 & 25 & 10 & 1 & 71,79 & 23,08 & 75,00 & 35,29 & 71,43 \\
\hline & & SCO & 2 & 31 & 7 & 2 & 78,57 & 22,22 & 50,00 & 30,77 & 81,58 \\
\hline & & WS-SF & 3 & 24 & 9 & 1 & 72,97 & 25,00 & 75,00 & 37,50 & 72,73 \\
\hline \multirow{3}{*}{06} & \multirow{3}{*}{15} & WS & 10 & 9 & 10 & 5 & 55,88 & 50,00 & 66,67 & 57,14 & 47,37 \\
\hline & & SCO & 9 & 2 & 14 & 6 & 35,48 & 39,13 & 60,00 & 47,37 & 12,50 \\
\hline & & WS-SF & 13 & 3 & 3 & 2 & 76,19 & 81,25 & 86,67 & 83,87 & 50,00 \\
\hline \multirow{3}{*}{07} & \multirow{3}{*}{15} & WS & 11 & 7 & 6 & 4 & 64,29 & 64,71 & 73,33 & 68,75 & 53,85 \\
\hline & & $\mathrm{SCO}$ & 10 & 5 & 12 & 5 & 46,88 & 45,45 & 66,67 & 54,05 & 29,41 \\
\hline & & WS-SF & 14 & 6 & 4 & 1 & 80,00 & 77,78 & 93,33 & 84,85 & 60,00 \\
\hline \multirow{3}{*}{08} & \multirow{3}{*}{02} & WS & 1 & 10 & 12 & 1 & 45,83 & 7,69 & 50,00 & 13,33 & 45,45 \\
\hline & & $\mathrm{SCO}$ & 1 & 9 & 7 & 1 & 55,56 & 12,50 & 50,00 & 20,00 & 56,25 \\
\hline & & WS-SF & 2 & 8 & 6 & 0 & 62,50 & 25,00 & 100,00 & 40,00 & 57,14 \\
\hline \multirow{3}{*}{\multicolumn{2}{|c|}{ Average }} & \multicolumn{5}{|l|}{ WS } & 60,51 & 31,17 & 62,27 & 39,49 & 57,89 \\
\hline & & \multicolumn{5}{|l|}{ SCO } & 56,07 & 28,85 & 48,71 & 35,01 & 53,52 \\
\hline & & \multicolumn{5}{|l|}{ WS-SF } & 71,97 & 53,15 & 85,93 & 63,28 & 62,10 \\
\hline
\end{tabular}

motile spermatozoas in white color and all the rest (background and immotile spermatozoas) in black color. The motility rate is obtained by labeling and counting de white blobs in the motility image.

After showing the practical feasibility, we now focus on comparative study and statistical evaluation of our system. The true concentration and motility values are unknown for all videos in the used database. Moreover, even if we have a CASA system, we cannot take its results as a basis for comparison. For this, we demanded three experienced andrologist experts to measure manually the two values (concentration and motility) for each of the 8 video sequences of the available database. The reference sperm cells are considered those marked by the majority of experts and they are utilized for comparaison for comparison in the evaluation stage to detect and compute the errors.

The obtained results for the manual analysis of the three experts and those of our system are presented in the Table 3 and Figure 9 for concentration, and in the Table 4 and Figure 10 for motility.

In Tables 3 and 4, we added two columns concerning the manual analysis results of the 3 operators to define the statistical values of the mean $\mu$ and the standard deviation $\sigma$. We have 
Figure 7. (a, a', a") Blobs labeling and ellipse drawing (b, b', b") After blobs classification (the spermatozoids are in blue color and debris in red color
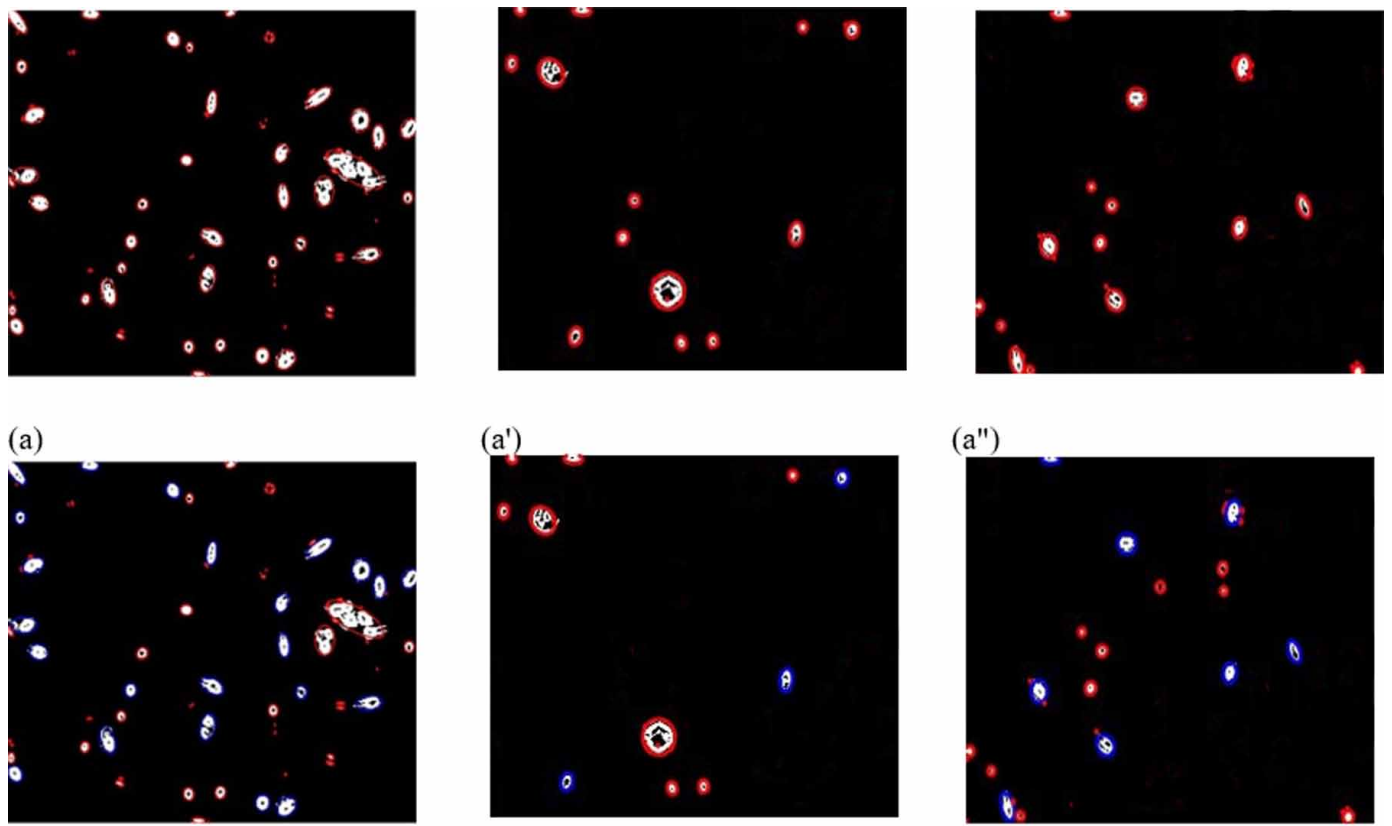

(b)

$\left(b^{\prime}\right)$

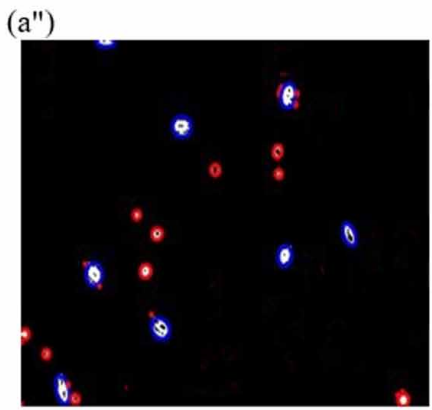

(b")

Figure 8. (a), (b) and (c) The 3 successive images Ik-1, lk, and lk+1 (d) and (e) The differential images dk and dk+1 (f) The motility image

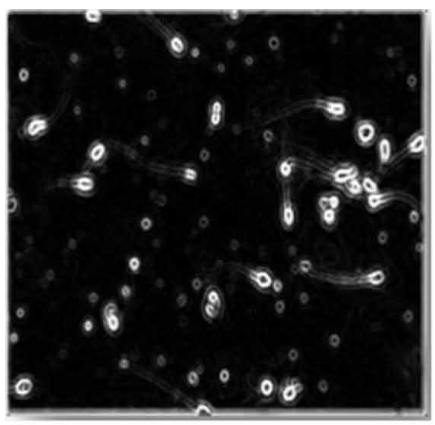

(a)

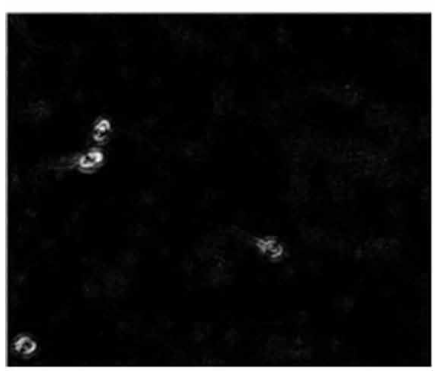

(d)

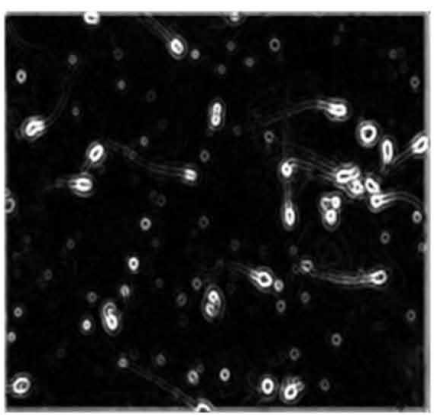

(b)

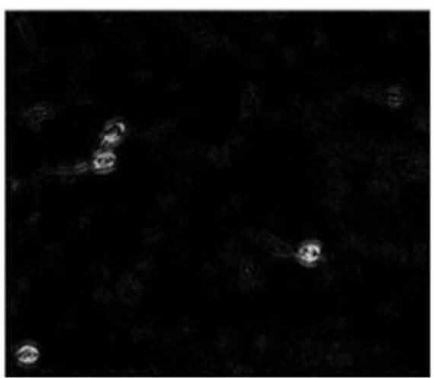

(e)

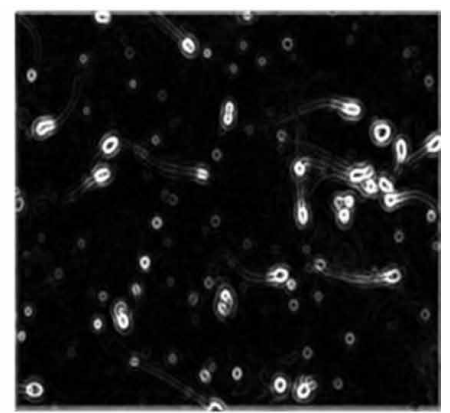

(c)

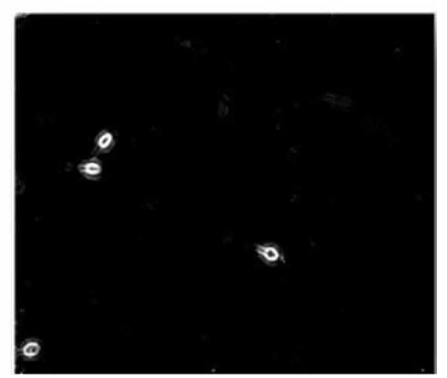

(f) 
Table 3. Obtained values of concentration by the 3 experts (manual method) and the proposed system (automatic method)

\begin{tabular}{|c|c|c|c|c|c|c|}
\hline \multirow[b]{2}{*}{ Videos } & \multicolumn{5}{|c|}{ Concentration - Manual method } & \multirow{2}{*}{$\begin{array}{c}\text { Concentration } \\
\text { Automatic } \\
\text { method } \\
\text { (proposed } \\
\text { system) }\end{array}$} \\
\hline & $\begin{array}{c}\text { Operator } \\
1\end{array}$ & $\begin{array}{c}\text { Operator } \\
2\end{array}$ & $\begin{array}{c}\text { Operator } \\
3\end{array}$ & $\begin{array}{c}\text { Mean } \\
\text { Value } \\
\mu_{c}\end{array}$ & $\begin{array}{c}\text { Standard } \\
\text { Deviation } \\
\sigma_{c}\end{array}$ & \\
\hline Video \# 1 & 02 & 03 & 03 & 3 & 1 & 02 \\
\hline Video \# 2 & 18 & 17 & 16 & 17 & 1 & 17 \\
\hline Video \# 3 & 04 & 05 & 06 & 5 & 1 & 05 \\
\hline Video \# 4 & 04 & 04 & 03 & 4 & 1 & 03 \\
\hline Video \# 5 & 05 & 04 & 04 & 4 & 1 & 03 \\
\hline Video \# 6 & 13 & 17 & 15 & 15 & 2 & 15 \\
\hline Video \# 7 & 13 & 15 & 16 & 15 & 2 & 16 \\
\hline Video \# 8 & 01 & 02 & 02 & 2 & 1 & 03 \\
\hline
\end{tabular}

Figure 9. Graphical representation of the Obtained values of concentration by the 3 experts (manual method) and the proposed system (automatic method)

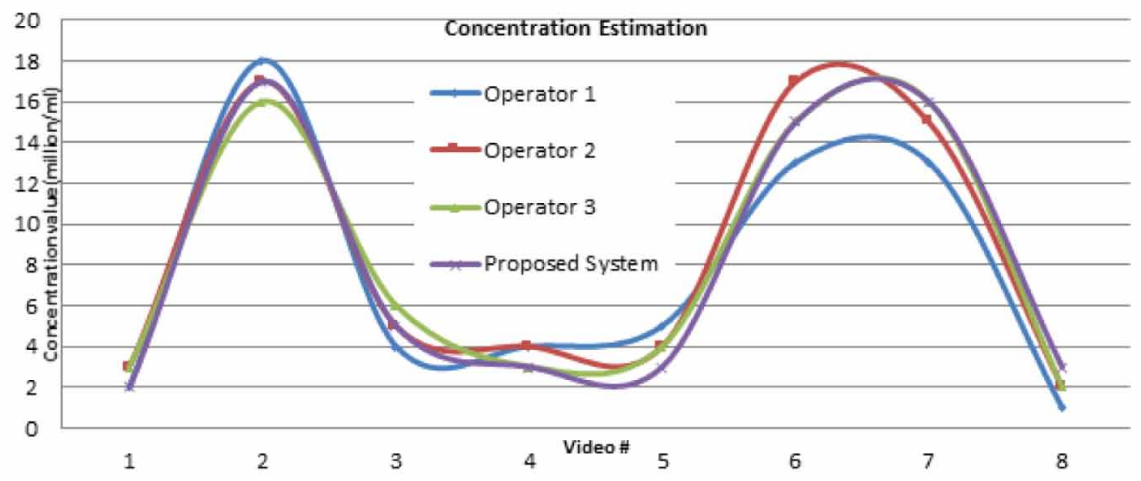

rounded them because they represent spermatozoids (whole numbers). After examining Table 3 and Figure 9, we noticed that the concentration values obtained with our system (automatic method) for the 8 video sequences are very precise and close to those obtained manually and are all in the interval: $\mu \mathrm{c} \pm \sigma \mathrm{c}$ of the manually measured value. We can confirm this graphically in Figure 9 and see that the curve representing the automatic concentration measurements is enveloped by the 3 manual measurements curves to $\pm \sigma \mathrm{c}$ near. Also, after examining Table 4 and Figure 10, we noticed that the motility values obtained with our system (automatic method) for the 8 video sequences are very precise and close to those obtained manually and are all in the interval: $\mu \mathrm{m} \pm \sigma \mathrm{m}$ of the manually measured value. We can confirm this graphically in Figure 10 and see that the curve representative of the automatic motility measurements is enveloped by the 3 manual measurements to $\pm \sigma$ m near.

We can therefore conclude that the proposed system gives very good results compared to those obtained manually. 
Table 4.Obtained values of motility by the 3 experts (manual method) and the proposed system (automatic method)

\begin{tabular}{|c|c|c|c|c|c|c|}
\hline \multirow[b]{2}{*}{ Videos } & \multicolumn{5}{|c|}{ Motility - Manual method } & \multirow{2}{*}{$\begin{array}{c}\text { Motility } \\
\text { Automatic } \\
\text { method } \\
\text { (proposed } \\
\text { system) }\end{array}$} \\
\hline & $\begin{array}{c}\text { Operator } \\
1\end{array}$ & $\begin{array}{c}\text { Operator } \\
2\end{array}$ & $\begin{array}{c}\text { Operator } \\
3\end{array}$ & $\begin{array}{c}\text { Mean } \\
\text { Value } \\
\mu \mathrm{m}\end{array}$ & $\begin{array}{c}\text { Standard } \\
\text { Deviation } \\
\sigma \mathrm{m}\end{array}$ & \\
\hline Video \# 1 & 01 & 02 & 01 & 1 & 1 & 01 \\
\hline Video \# 2 & 05 & 04 & 06 & 5 & 1 & 06 \\
\hline Video \# 3 & 03 & 04 & 05 & 4 & 1 & 04 \\
\hline Video \# 4 & 01 & 01 & 03 & 2 & 1 & 01 \\
\hline Video \# 5 & 03 & 03 & 03 & 3 & 0 & 03 \\
\hline Video \# 6 & 07 & 13 & 12 & 11 & 3 & 12 \\
\hline Video \# 7 & 08 & 07 & 10 & 8 & 2 & 09 \\
\hline Video \# 8 & 01 & 01 & 01 & 1 & 0 & 01 \\
\hline
\end{tabular}

Figure 10. Graphical representation of the Obtained values of motility by the 3 experts (manual method) and the proposed system (automatic method)

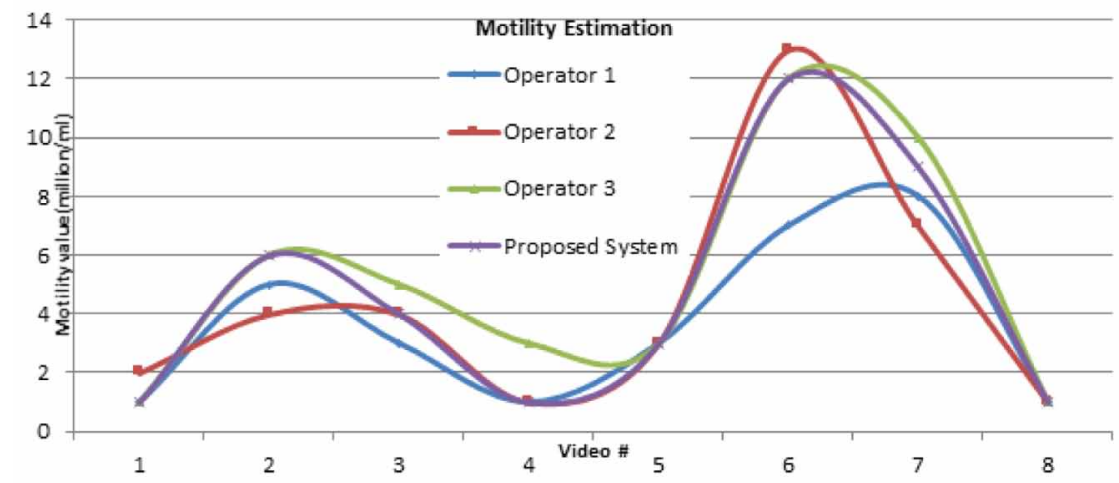

\section{CONCLUSION}

In this paper, a technique of automatic assessment of spermatozoa concentration and motility is presented. It begins by the pretreatment of the human sperm microscopic videos which consists of a conversion of the RGB color space into the YCbCr space, the "Gaussian filtering" and the "discrete wavelet filtering". Followed by the segmentation of the image into two classes: spermatozoas and the background (noise and debris). To achieve this, we used a reinforcing technique of two types of segmentation, one based on "watershed" and the other based on "Sobel Contour detector". Furthermore, It uses a machine learning technique that consist on 2 classes classification based on invariant characteristics that are the dimensions of the bounding ellipse of the spermatozoa head as well as its surface to separate true sperm from false ones. The final step was devoted to the detection of motile spermatozoas. This was done by the inter-frame difference technique performed on three successive images.

To evaluate the performance of the proposed algorithm, it was examined on real microscopic videos. Then, the obtained results were compared with those performed manually by three andrologists for motility and concentration assessment. The segmentation results were compared with two other 
existing methods (i.e., WS and Sobel). The comparisons showed the superiority of the proposed method to its alternatives. This means that the proposed algorithm distinguished sperms from other semen parts and it can be concluded that the proposed method can be used as a suitable choice for detecting sperms in microscopic images captured from human semen. As future work, we intend to compare it with commercialized CASA systems and to increase the number of andrologists in order to obtain a more important basis of comparison. We also plan to expand our system to perform other sperm assessment such as sperm morphology analysis, motion analysis. This will allow us to offer the end user a universal CASA system.

\section{ACKNOWLEDGMENT}

The authors would like to thank Mr. Mohammad Reza AHMADZADEH, PhD, Isfahan University of Technology (Iran) for allowing us to use his database of microscopic sperm videos and his manual assessment of the used videos. We would like to thank Dr N. BENAMAR and Dr. A. BOUALEM, Andrologists at EHU Hospital of Oran (Algeria) for helping us to do the manual assessment of sperm motility from the videos and their valuable remarks and suggestions. 


\section{REFERENCES}

Abbiramy, V. S., Shanthi, V., \& Allidurai, C. 2010, Spermatozoa detection, counting and tracking in video streams to detect asthenozoospermia. IEEE International Conference on Signal and Image Processing (ICSIP), 265-270. doi:10.1109/ICSIP.2010.5697481

Amann, R. P., \& Waberski, D. (2014). Computer-assisted sperm analysis (CASA): Capabilities and potential developments. Theriogenology, 81(1), 5-17. doi:10.1016/j.theriogenology.2013.09.004 PMID:24274405

Arkanfari, R., \& Shojaedini, S. V. (2018). A New Method for Detecting Sperms in Microscopy Images: Combination of Zernike Moments and Spatial Processing. Iranian Journal of Medical Physics, 15(4), $215-221$.

Bijar, A., Benavent, A. P., Mikaeili, M., \& khayati, R. (2012). Fully automatic identification and discrimination of sperm's parts in microscopic images of stained human semen smear. Journal of Biomedical Science and Engineering, 5(7), 384-395. doi:10.4236/jbise.2012.57049

Cooper, T. G., Noonan, E., von Eckardstein, S., Auger, J., Baker, H. W. G., Behre, H. M., Haugen, T. B., Kruger, T., Wang, C., Mbizvo, M. T., \& Vogelsong, K. M. (2010). World Health Organization reference values for human semen characteristics. Human Reproduction Update, 16(3), 231-245. doi:10.1093/humupd/dmp048 PMID:19934213

Gang, L., Shangkun, N., \& Yugan, Y. (2013). An improved moving objects detection algorithm, IEEE International Conference on Wavelet Analysis and Pattern Recognition (ICWAPR), 96-102.

Gao, W., Zhang, X., \& Yang, L. (2010). An improved Sobel edge detection. The 3rd IEEE International Conference on Computer Science and Information Technology (ICCSIT), 67-71.

Ghasemian, F., Mirroshandel, S. A., Monji-azad, S., Azarnia, M., \& Zahiri, Z. (2015). An efficient method for automatic morphological abnormality detection from human sperm images. Computer Methods and Programs in Biomedicine, 122(3), 409-420. doi:10.1016/j.cmpb.2015.08.013 PMID:26345335

Grover, T. (2016). Denoising of medical images using wavelet transform. Imperial Journal of Interdisciplinary Research, 2(3), 541-548.

Illustration-stock-anatomie-de-spermatozoid. (2018). https://fr.dreamstime.com/illustration-stock-anato mie-despermatozo\%C3\%AFde-image 56678357.

Imani, Y., Teyfouri, N., \& Ahmadzadeh, M. R. (2014). A new method for multiple sperm cells tracking. Journal of Medical Signals and Sensors, 4(1), 35. doi:10.4103/2228-7477.128436 PMID:24696807

Khalifa, Taha, \& Hassanien. (2018). Automatic Counting and Visual Multi-tracking System for Human Sperm in Microscopic Video Frames. In International Conference on Advanced Intelligent Systems and Informatics. Springer.

Mahdavi, H. S., Monadjemi, A., \& Vafae, A. (2011). Sperm detection in video frames of semen sample using morphology and effective ellipse detection method. Journal of Medical Signals and Sensors, 1(3), 206. doi:10.4103/2228-7477.95392 PMID:22606677

Mustafa, N., Khan, S. A., \& Li, J. (2014). Medical image De-noising schemes using wavelet transform with fixed form thresholding. IEEE 11th International Computer Conference on Wavelet Active Media Technology and Information Processing (ICCWAMTIP), 397-402.

Nallella, K. P., Sharma, R. K., Aziz, N., \& Agarwal, A. (2006). Significance of sperm characteristics in the evaluation of male infertility. Fertility and Sterility, 85(3), 629-634. doi:10.1016/j.fertnstert.2005.08.024 PMID:16500330

NormsO. M. S. (2010). http://www.aly-abbara.com/echographie/biometrie/scores/spermogramme_normes_oms. html

Otsu, N. (1979). A threshold selection method from gray-level histograms. IEEE Transactions on Systems, Man, and Cybernetics, 9(1), 62-66. doi:10.1109/TSMC.1979.4310076

Ravanfar, M. R., \& Moradi, M. H. (2011). Low contrast sperm detection and tracking by watershed algorithm and particle filter. The 18th IEEE Iranian Conference of Biomedical Engineering (ICBME), 260-263. 
Shaker, F., Monadjemi, S. A., \& Naghsh-nilchi, A. R. (2016). Automatic detection and segmentation of sperm head, acrosome and nucleus in microscopic images of human semen smears. Computer Methods and Programs in Biomedicine, 132, 11-20. doi:10.1016/j.cmpb.2016.04.026 PMID:27282223

Shojaedini, S. V., Goldar, A. R., \& Soori, M. (2017). Correntropy based sperm detection: A novel spatiotemporal processing for analyzing videos of human semen. Health and Technology, 1-8.

Syahputra, M. F., Chairani, R., Seniman, , Rahmat, R. F., Abdullah, D., Napitupulu, D., Setiawan, M. I., Albra, W., Erliana, C. I., \& Andayani, U. (2018). Identification Male Fertility Through Abnormalities Sperm Based Morphology (Teratospermia) using Invariant Moment Method. Journal of Physics: Conference Series, 978(1), 012107. doi:10.1088/1742-6596/978/1/012107

Urbano, L. F., Masson, P., Vermilyea, M., \& Kam, M. (2017). Automatic tracking and motility analysis of human sperm in time-lapse images. IEEE Transactions on Medical Imaging, 36(3), 792-801. doi:10.1109/ TMI.2016.2630720 PMID:27875219

Karima Boumaza received the B.S. degree in Mathematics and computer engineering from Moulay Tahar University, Saida, Algeria, in 2010, and the M.S degrees in computer engineering from the same university, in 2012. She has been a Researcher in Intelligent Systems Research Laboratory- LARESI- laboratory, University of Science and Technology of Oran - USTO, Algeria since 2012. Her research interests include image processing, medical imaging, object tracking, pattern recognition, motion detection, and noise reduction.

Abdelhamid Loukil obtained his PhD at the University of Evry Val d'Essonne (France) in 1993. He is currently Professor in the Electrical Engineering Faculty of the University of Science and Technology of Oran - USTO (Algeria) where he teaches computer sciences, image processing and robot vision. He is a member of the LARESI laboratory and head of the "Mobile Robotics and Vision" research team. His research interests include image processing, artificial vision, artificial intelligence, robotics and human-machine interface design. 